\title{
CORRECTION
}

\section{Correction to: Hiatal hernia, mitral valve prolapse and defecatory disorders: An underlying rectal prolapse?}

\author{
M. Shalaby ${ }^{1,3} \cdot$ P. Polisca $^{2} \cdot$ G. Missori ${ }^{1} \cdot$ P. Sileri $^{1}$
}

Published online: 15 October 2018

(c) Springer Nature Switzerland AG 2018

\section{Correction to: Tech Coloproctol (2016) 20:337-338 https://doi.org/10.1007/s10151-016-1442-6}

Unfortunately, the 2nd affiliation of Dr. M. Shalaby has been missed out in the original publication. The complete 2 nd affiliation of the same is given below.

Department of General Surgery, Mansoura University, Mansoura, Egypt.

The original article can be found online at https://doi.org/10.1007/ s10151-016-1442-6.

P. Sileri

piersileri@yahoo.com

1 Department of General Surgery, Policlinico Tor Vergata di Roma UOC B, University of Rome Tor Vergata, Viale Oxford 81, 00133 Rome, Italy

2 Department of Internal Medicine, Policlinico Tor Vergata, University of Rome Tor Vergata, Viale Oxford 81, 00133 Rome, Italy

3 Department of General Surgery, Mansoura University, Mansoura, Egypt 